\title{
Análisis sísmico del noroeste de San Rafael del Sur
}

Tupak Ernesto Obando Rivera, geólogo-investigador

Instituto Nicaragüense de Estudios Territoriales, tobando_geologic@yahoo.com

\section{RESUMEN}

La investigación analiza la sismicidad del Noroeste de San Rafael del Sur para ordenamiento físico de ese territorio. En el estudio realizado, se revisaron recursos bibliográfico y cartográfico existentes; se interpretaron modelos geo-estructurales del terreno; se calcularon e interpretaron datos geológicos de campo usando equipos electrónicos; se analizaron sismos histórico - instrumentales con software ArcGis 9. Con esto se obtuvo cartografía sísmica y geológica del área estudiada. A su vez, se logró la zonación sísmica usando modelo numérico

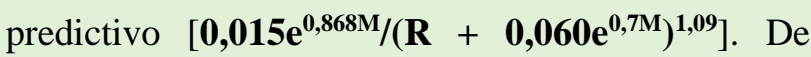
igual manera, se obtuvo geometría espacial de fuentes sismo-generadoras locales. Asimismo, se lograron perfiles de suelos detallados. Finalmente, se alcanzó la iconografía pormenorizada de características geológicas in situ. A partir de los resultados obtenidos, se concluye que fallas geológicas ocultas constituyen la generatriz de sismos, expresada en 24 eventos superficiales menor de $8.9 \mathrm{~km}$ y magnitudes entre 2,7 a $3,3^{\circ}$ Ritcher, formando 3 ejes de dirección SuroesteNoreste y Norte-Sur vistos en terrazas de Ríos Jesús y Jordán. Finalmente, se revela suelos frágiles ante intensidad sísmica entre 0.4 - $0.5 \mathrm{~g}$ en El Tamarindo y Los Jaras, contrario a suelos arenosos firmes y estables de San Rafael del Sur con efecto atenuador de 0,009 a $0,01 \mathrm{~g}$

Palabras Clave.- sismo, geología, generatriz, magnitud

\section{SUMMARY.-}

The research analyzes the seismicity of Northwest San Rafael del Sur for physical planning of the territory. In the study, bibliographic and cartographic existing resources were reviewed; geo-structural models were interpreted terrain; They were calculated and interpreted geological field data using electronic equipment; historical earthquakes were analyzed - instrumental software ArcGis 9. With this seismic and geological mapping of the area studied was obtained. In turn, the seismic zonation was achieved using predictive numerical model [0,015e0,868M / (R + 0,060e0,7M) 1.09]. Similarly, spatial geometry of earthquake-generating local sources was obtained. Also, detailed soil profiles were achieved. Finally, the iconography detailed geological characteristics in situ was reached. From the results, it is concluded that hidden faults constitute the generatrix of earthquakes, surface expressed in 24 events less than $8.9 \mathrm{~km}$ and magnitudes between 2.7 to $3.3^{\circ}$ Richter, forming 3axis direction and Southwest-Northeast North-South River terraces seen in Jesus and Jordan. Finally, fragile soils is revealed to seismic intensity between $0.4-0.5 \mathrm{~g}$ in El Tamarindo and Los Jaras, contrary to firm and stable sandy soils of San Rafael del Sur with attenuating effect of 0.009 to $0.01 \mathrm{~g}$

Keywords .- words earthquake, geology, generatrix magnitude 


\section{INTRODUCCIÓN}

Esta investigación se enmarca en un área al noroeste de San Rafael del Sur, a sólo 46km de Managua cuyo objetivo es analizar la sismicidad para la planificación física, y uso importante de esa región. Algunos trabajos realizados por GSHAP (1999), considera la zona con alta amenaza sísmica, e importante por su proximidad a la estructura activa Graben de Managua, en donde residen 41,574 habitantes $\left(116 \mathrm{hab} / \mathrm{km}^{2}\right)$. El sitio investigado con forma rectangular y superficie de $46 \mathrm{~km}^{2}$ (Ilustración 1), limitó al Suroeste con Masachapa; al Norte de San Cayetano; al Este con Casa de Teja; y al Sur con San Rafael del Sur y La Junta

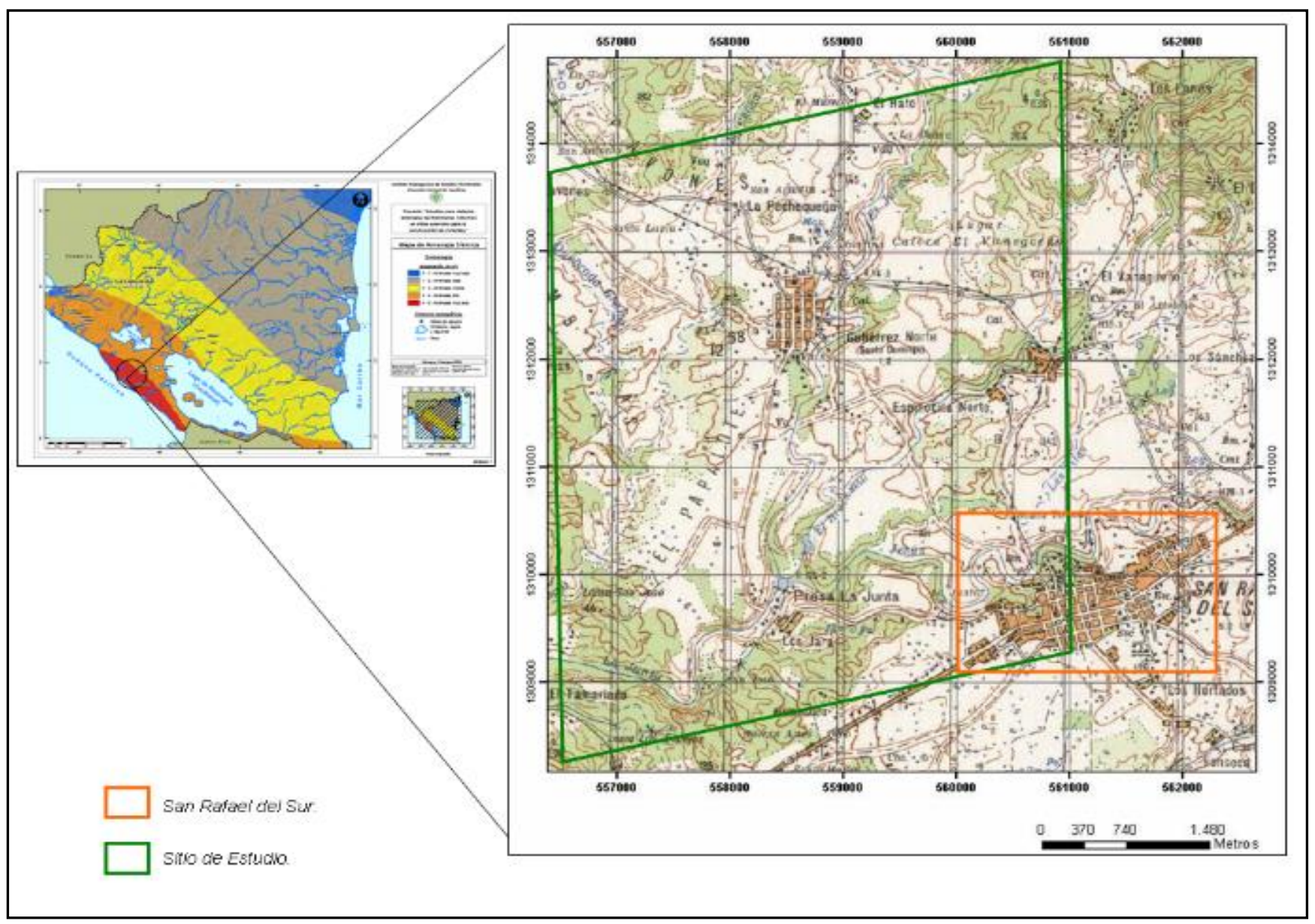

Figura 1. Localización del área estudiada a escala 1:37,000. Fuente: IGN, 1971

El territorio de interés fue propuesto por la disponibilidad de datos de sismos superficiales locales, un área de interés económico y turístico, y fácilmente accesible en vehículo, en cuyos 
alrededores se emplazan obras civiles horizontales y verticales de carácter residencial, comercial, educacional e industrial importante para sus pobladores y comunidades vecinas.

\section{MATERIAL Y MÉTODO}

La presente investigación es tipo descriptivo - analítico, de carácter retrospectivo y de corte transversal. El territorio investigado está ubicado al Noroeste de San Rafael del Sur según Mapa topográfico del lugar con código 2951-IV del año 1986 a escala 1:50,000 publicado por el Instituto Nicaragüense de Estudios Territoriales (INETER) (figura 1)

Para esto se aplicó técnicas informática, y documentales iconográficas, la observación y medición de campo usando equipos electrónicos, modelos matemáticos, y cartográficos a escala 1/50,000

Para obtener la información se procedió de la siguiente manera:

\section{Revisión bibliográfica y fuentes de documentación}

Se revisó y analizaron recursos bibliográficos disponibles en instituciones públicas del país y consultas a expertos nacionales. La bibliografía consultada se encontró en Biblioteca Jaime Incer Barquero en Managua

\section{Equipo de Oficina.}

Los instrumento empleados en tarea de oficina fueron accesorios de escritorio (borrador, lápices de colores, papelería, lápiz y borrador), mapas topográfico y geológico a escala 1:50,000; documentos escritos; uso e impresiones de computadora de la biblioteca.

\section{Trabajo de campo}

Esta etapa se buscó evidencias físicas en el terreno (figura 2) de la actividad sísmica debido a fuente sismo-generadora locales, aprovechando las condiciones de accesibilidad de los lugares como El Tamarindo, Los Jaras y terrazas fluviales de Río Jordan y Jesús.

\section{Equipo de campo}

Los instrumentos de campo empleados fueron martillo de geólogo, cinta métrica, lápiz y borrador, libreta de campo, cámara fotográfica y rollos, GPS manual marca GARMIN eTrex Summit, fotografías aéreas, y otros. 
Para comprobar la medición de parámetros y ubicación de elementos de interés geológico en sitios puntuales del área investigada se han tomado fotografía con cámara digital de 4.0 megapixeles de resolución marca OLYMPUS

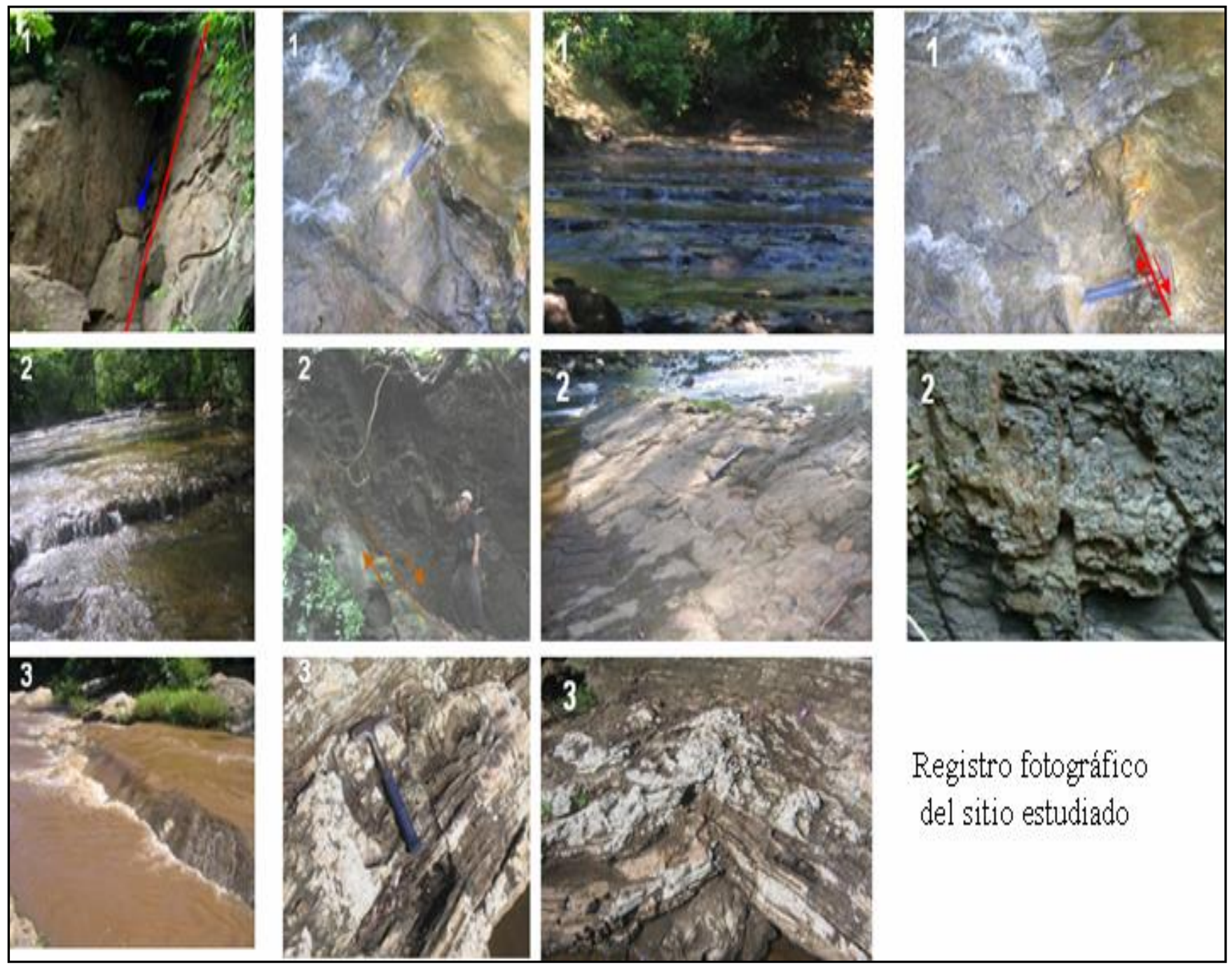

Figura 2 documentación iconográfica local

\section{Procesamiento y análisis de la información}

En esta fase se presentaron gráficamente los resultados a través de mapas, gráfico y otros. Se preparó cartografía de detalle usando programa informático del sistema de información geográfica (SIG) como ArcGis 9.

A su vez, se empleó programas de Microsoft Office como Word y Excel para la elaboración del documento final. 


\section{Elaboración del informe}

Esta fase consistió en la elaboración de un documento final siguiendo pasos antes mencionado, obtenidos del trabajo geológico de campo y tareas de oficina con sus respectivos mapas, gráficos, diagramas, análisis de la situación, conclusiones y recomendaciones.

\section{RESULTADOS Y DISCUSIÓN}

Los resultados del mapa (figura 3) de epicentros y estructuras geológicas para el área Noroeste de San Rafael del Sur, presenta áreas con actividad tectónica alta en comunidades Tamarindo, Los Jaras y Gutiérrez Norte. Estos lugares están alojados en tres planos de esfuerzo sísmico, dos de dirección Noreste-Suroeste y uno Norte-Sur. En el área de estudio se señalan epicentros de sismos con magnitudes de 0 a $3{ }^{\circ}$ Ritcher con profundidades $0-3 \mathrm{~km}$, capaz de ocasionar menoscabos en viviendas locales exigua calidad sismo-resistente debido a materiales empleados y tipología constructiva.

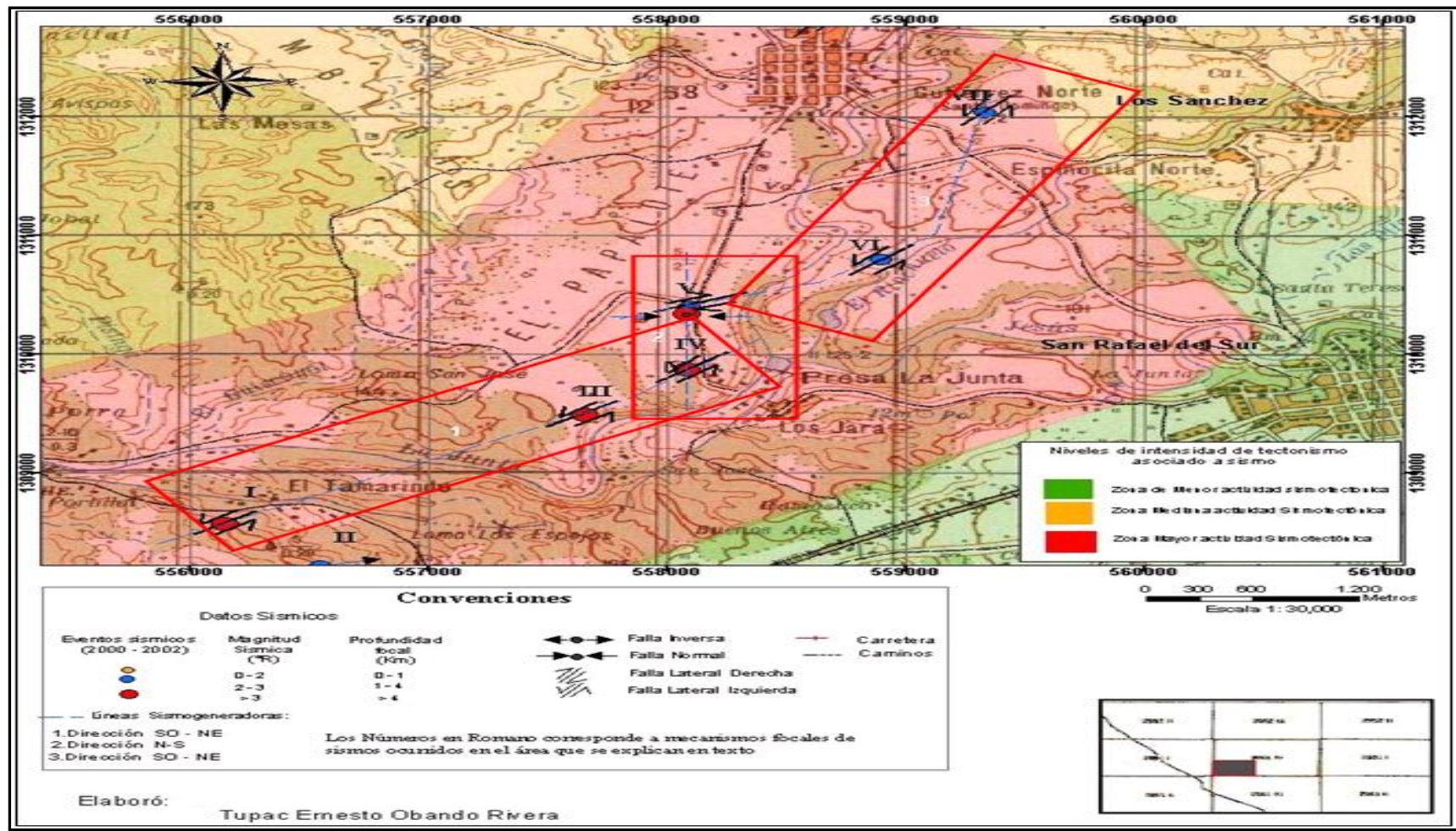

Figura 3. Mapa epicentral y elementos geológicos 
En el territorio investigado, se presentan dos tipos de roturas en el terreno (figura 4) acompañados de desplazamientos en el suelo (fallas geológicas) diferenciada una de otras por sus componentes de movimiento, uno de tipo vertical y otro horizontal con evidencia impresa en afloramientos rocoso y terrazas fluviales de Ríos Jesús y Jordán

Estos primeros resultados confirman lo enunciado por otros especialistas como Segura (2005), quién valora San Rafael del Sur y sus alrededores como zona de baja sismicidad superficial con más de 30 años de inactividad. Segura, sugiere un comportamiento anómalo reconocido instrumentalmente entre los años 2000-2002.

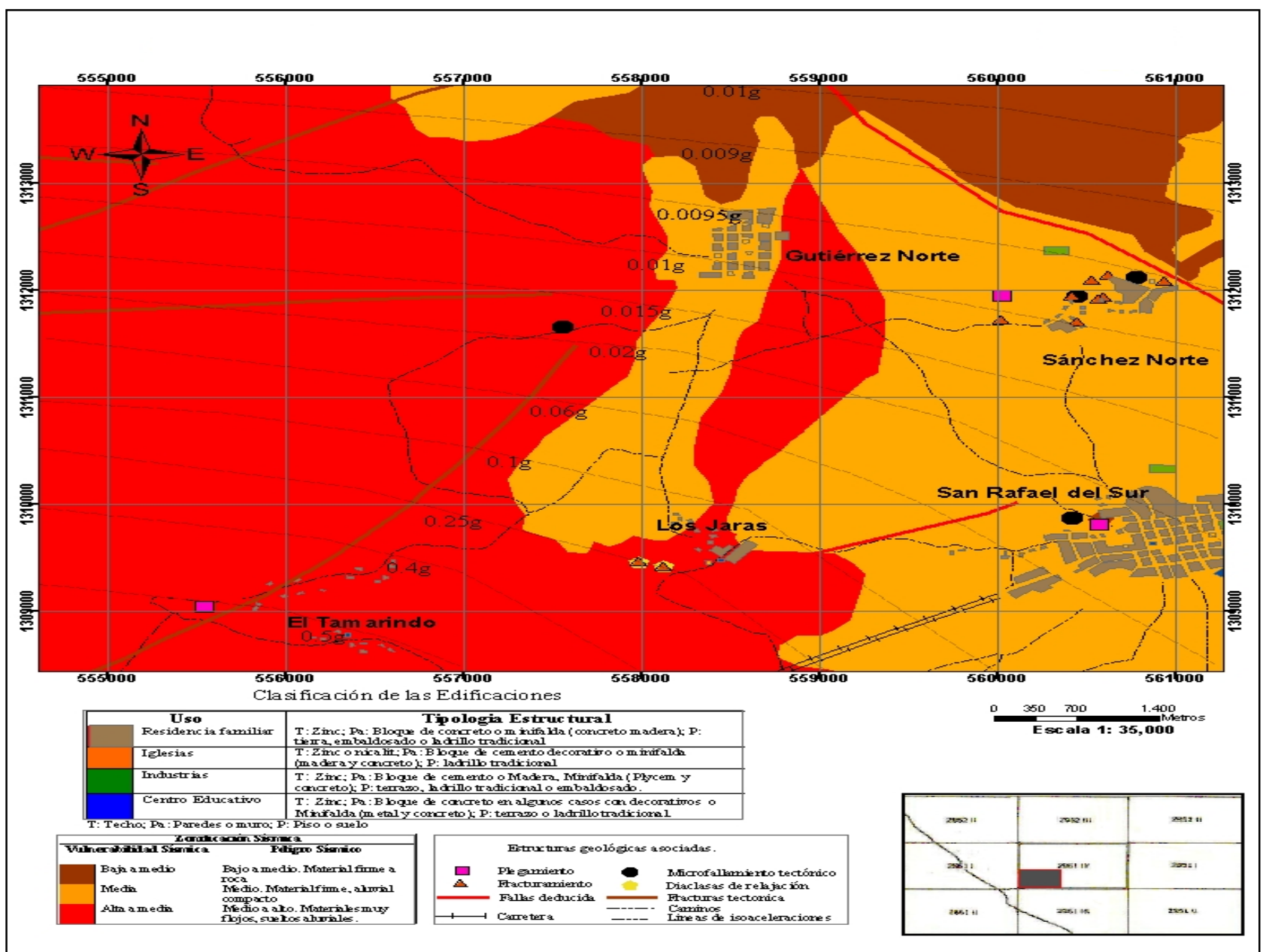

Figura 4. Zonación sísmica local aplicando modelo numérico: $\left[0,015 \mathrm{e}^{0,868 \mathrm{~m}} /\left(\mathrm{r}+0,060 \mathrm{e}^{0,7 \mathrm{~m}}\right)^{1,09}\right]$

De acuerdo con el mapa de zonificación sísmica se señalan valores de intensidad sísmica alto sobre todo en la comunidad El Tamarindo y Los Jaras, en donde la consistencia del suelo blando y frágiles comprendido entre 0,4 a 0,5 grados de gravedad (g). Y mediciones baja en la intensidad del movimiento 
sísmico, particularmente, en la municipalidad de San Rafael del Sur con valores entre 0,009 a 0,01 grados de gravedad ${ }^{1}(\mathrm{~g})\left({ }^{1} \mathrm{~g}\right.$ : Componente vertical de la aceleración del suelo igual a $980 \mathrm{~cm} / \mathrm{s}^{2)}$ debido a la consistencia física y consistencia de suelo firmes y estables.

De igual manera, se presentan en el área de interés evidencias físicas impresas en rocas, tales como fracturas y flexuras de material geológico, deslizamiento, microfallamiento tectónico, entre otros.

A su vez, se presenta en el lugar de estudio edificaciones físicas, ocupadas mayormente por viviendas familiares construidas con techo de zinc, paredes de bloque de concreto o minifalda (concreto de manera) y piso de tierra, embaldosado o ladrillado tradicional.

Por el contrario, algunos expertos, sugieren sismos de foco superficial con intensidades sísmica menores entre 0,05 a 0,24 grados de gravedad (g) para San Rafael del Sur comprendida entre con períodos de retorno entre 5 a 500años. (Zapata R, 1985: 16)

Finalmente, se obtienen mecanismos de roturas del suelo a través de programa informático SUNSYSTEM X-WIN 32 para uso en ambiente sísmico con libre carácter de distribución en INTERNET. Los resultados gráficos (Ilustraciones $\mathbf{5 , 6 , 7 , 8 , 9}$ y 10) y su análisis se presentan a continuación A.- Sugiere un sismo con magnitud de $3.5^{\circ}$ Ritcher, profundidad focal de $100 \mathrm{~m}$ ocurrido a 05:24Hrs asociado a falla lateral izquierda obtenida del mecanismo de ruptura I. (Figura 5)

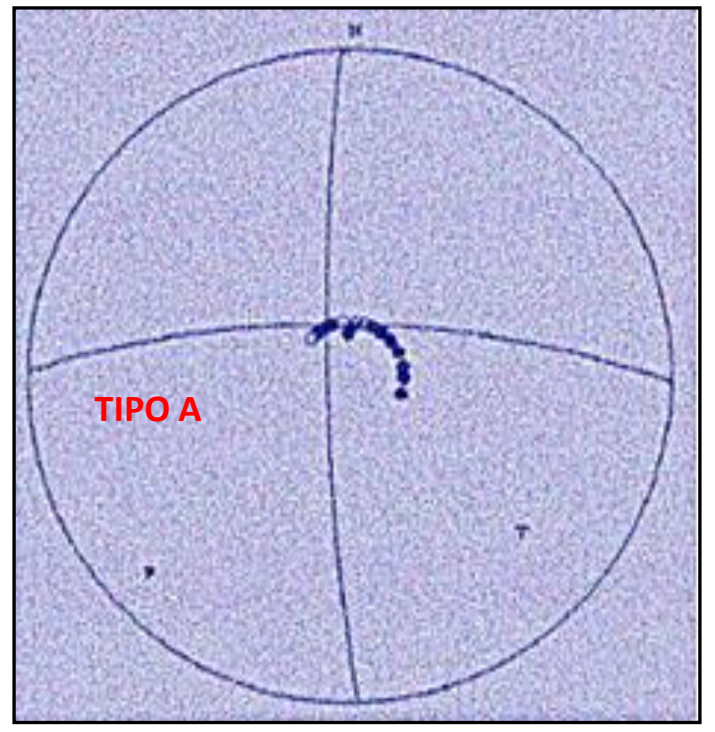

Figura 5. Mecanismo geométrico de rotura no 1. 


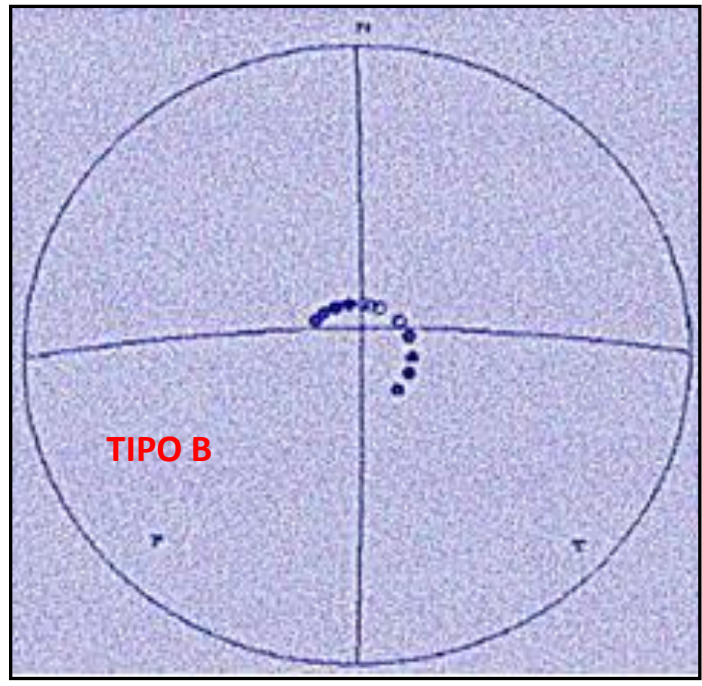

B.- Indica un sismo con magnitud de $3.0^{\circ}$ Ritcher, profundidad focal de $0 \mathrm{~m}$ ocurrido a 05:24Hrs asociado a falla lateral izquierda obtenida del mecanismo de ruptura III (Figura 6)

Figura 6. Mecanismo geométrico de rotura no 2

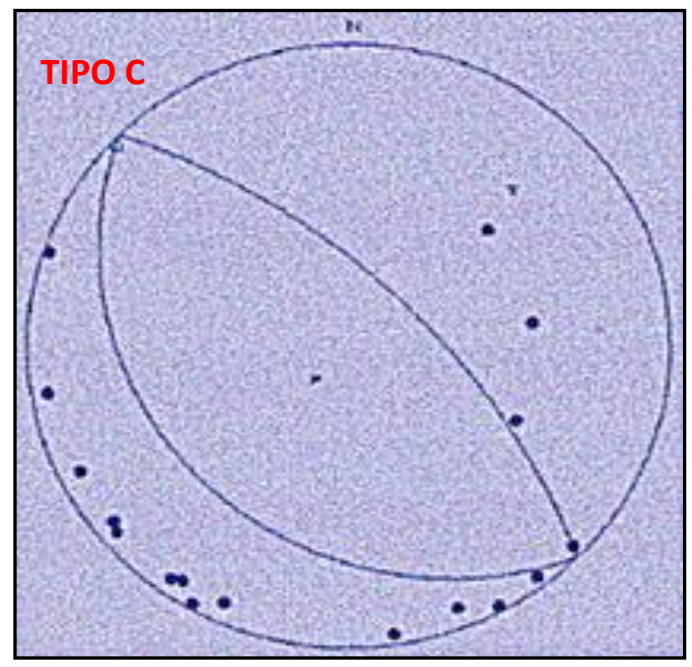

C.- Señala un sismo con magnitud de 2.6 Ritcher, profundidad focal de $8.5 \mathrm{~km}$ ocurrido a $05: 20 \mathrm{Hrs}$ asociada falla normal obtenida del mecanismo de ruptura IV. (Figura 7)

Figura 7. Mecanismo geométrico de rotura no 3 


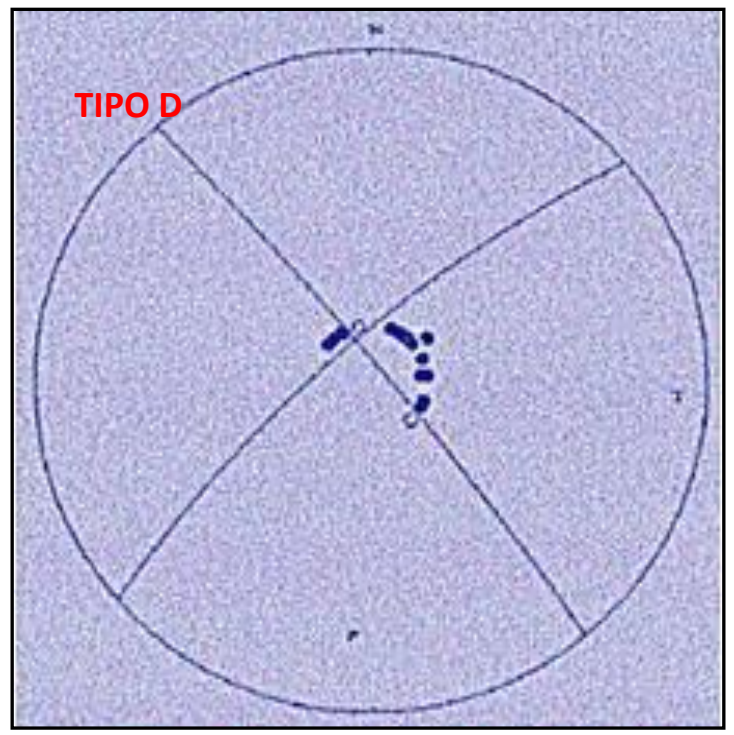

D.- Sugiere un sismo con magnitud de $2.8^{\circ}$ Ritcher, profundidad focal de $8 \mathrm{~km}$ ocurrido a $05: 20 \mathrm{Hrs}$ asociado a falla lateral derecha obtenido del mecanismo de ruptura $\mathrm{V}$ (Figura 8)

Figura 8. Mecanismo geométrico de rotura no 4

E.- Indica un sismo con magnitud de $2.5^{\circ}$ Ritcher, profundidad focal de $1.1 \mathrm{~km}$ ocurrido a $05: 20 \mathrm{Hrs}$ asociada falla lateral izquierda obtenida del mecanismo de ruptura VI (Figura 9)

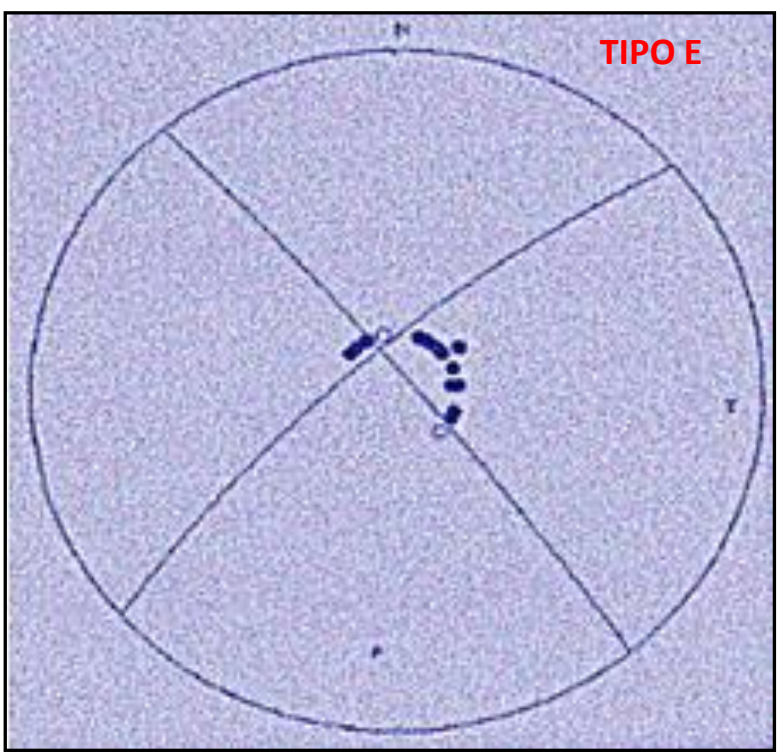

Figura 9. Mecanismo geométrico de rotura no 5 


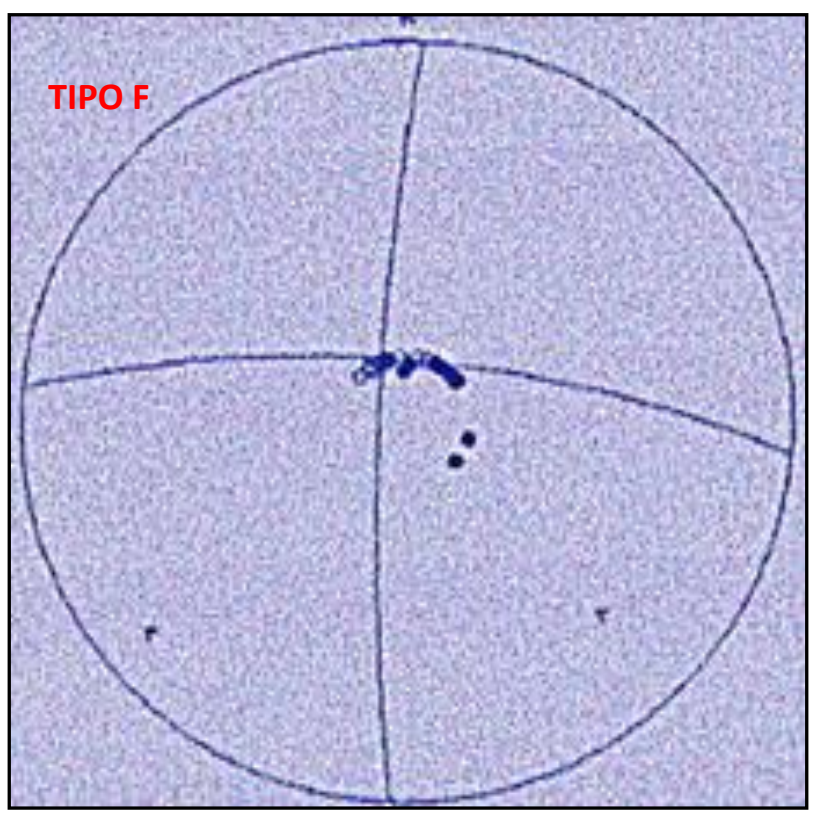

F.- Señala un sismo con magnitud de $3.6^{\circ}$ Ritcher, profundidad focal de $1 \mathrm{~km}$ ocurrido a 04:21Hrs asociado a falla lateral izquierdo obtenido del mecanismo de ruptura VII.

(Figura 10)

Figura 10. Mecanismo geométrico de rotura no 6

\section{CONCLUSIONES}

A partir del análisis de los resultados, se concluye que en área al noroeste de San Rafael del Sur:

La principal fuente sismo-generadora local reconocida lo constituye fallas geológicas con desplazamiento del suelo horizontal, pero sus vectores de movimiento superficial escapan de la observación en el terreno.

Veinticuatro sismos perpendiculares recientes conforman dos planos de tendencia, dos Suroeste-Noreste y uno Norte-Sur relacionado con hallazgo de actividad sísmica encontrados en afloramiento de rocas y terrazas de Ríos Jesús y Jordán.

Las profundidad de sismos particulares varían de $0-3 \mathrm{~km}$ con magnitudes entre $2-3{ }^{\circ}$ Ritcher.

La densidad ${ }^{2}$ de evidencia física en los alrededores del área de interés, es de $0.52 \mathrm{~km} / \mathrm{km}^{2}$ para fallas geológicas y $0.29 \mathrm{~km} / \mathrm{km}^{2}$ fracturas tectónicas.

Al aplicar modelos numérico predictivo $\left[\mathbf{0 , 0 1 5} \mathrm{e}^{\mathbf{0 , 8 6 8 M}} /\left(\mathbf{R}+\mathbf{0 , 0 6 0} \mathrm{e}^{\mathbf{0 , 7 M}}\right)^{\mathbf{1 , 0 9}}\right]$, se muestra suelos frágiles arcillo-limoso sueltos o aluviales sin consolidar ante intensidad sísmica entre 0.4 - $0.5 \mathrm{~g}$ en El Tamarindo y Los Jaras calificándose con niveles mayores de Peligro Sísmico, contrario a suelos de 
textura arenosa firmes y estables de San Rafael del Sur, Los Gutiérrez Norte y Sánchez Norte con efecto atenuador de 0,009 a 0,01g calificándose con niveles menores de Peligro Sísmico.

\section{AGRADECIMIENTO}

A Msc. Sismológo Fabio Segura Mójica, por su colaboración profesional en esta investigación.

\section{REFERENCIAS}

Instituto Geográfico Nacional (1971). Mapa geológico cuadrángulo "San Rafael del Sur, a escala 1:50,000”. Managua. Instituto Geográfico Nacional. $1^{\text {era }}$ edición.

Programa Global para la Amenaza Sísmica -GSHAP - (1999). Mapa de Amenaza Sísmica de Nicaragua a escala 1:1000,000. Managua. GSHAP. $1^{\text {era }}$ edición

Segura, F. (2005). Sismos en San Rafael del Sur (entrevista). Managua, Dirección General de Geofísica, INETER-Managua.

Zapata, R. y Duarte, M. (1985). Riesgo sísmico para “Ampliación de la planta de Cemento” en San Rafael del Sur. Managua. SGN. $1^{\text {era }}$ edición 